\title{
Habilidades de cuidado básico y la carga del cuidador familiar de pacientes con déficit neurológico en el servicio de hospitalización de adultos del Hogar Clínica San Juan de Dios, Lima
}

\author{
Sandra Vanessa Mamani-Napán ${ }^{1 *}$, Isabel Peñaloza-Rojas², Karem Vilca-Arteaga ${ }^{3}$ \\ ${ }^{1}$ Hospital de Baja Complejidad Vitarte - Av. Nicolás Ayllón 5880 - ATE \\ ${ }^{2}$ Hospital de Emergencias "José Casimiro Ulloa" - Av. Roosvelt N. ${ }^{\circ} 6355$ - Miraflores \\ ${ }^{3}$ Hospital Nacional Daniel Alcides Carrión - Guardia Chalaca 2176 - Bellavista
}

\begin{abstract}
RESUMEN
Objetivo: Determinar la relación entre las habilidades del cuidado básico y la carga del cuidador familiar en pacientes con déficit neurológico en el servicio de hospitalización adulto del Hogar Clínica San Juan de Dios. Lima, enero a marzo, 2012. Material y Métodos: Fue un estudio cuantitativo descriptivo y de diseño correlacional. La unidad de análisis fueron 60 pacientes hospitalizados de la Clínica San Juan de Dios, la información fue generada a través de 2 instrumentos, un cuestionario y una lista de verificación aplicados por el profesional de Enfermería. Resultados: El 67.5\% del cuidador familiar presentó habilidades en nivel deficiente, acompañado de una sobrecarga intensa del $73 \%$. Conclusiones: Se encontró relación inversa y significativa entre las habilidades del cuidador familiar y la carga del cuidador familiar, expresada en que a menor nivel de habilidades en el cuidado básico, más intensa es la sobrecarga que experimenta el cuidador familiar de pacientes con déficit neurológico en el servicio de hospitalización de adultos del Hogar Clínica San Juan de Dios $(p=0,028)$.
\end{abstract}

Palabras clave: Habilidades de cuidado básico- carga del cuidador familiar- pacientes adultos- déficit neurológico.

\begin{abstract}
Objective: To determine the relationship between basic care skills and family caregiver burden in patients with neurological deficit in the adult inpatient service Hogar Clínica San Juan de Dios. Lima, January to March, 2012. Material and Methods: The type of study was quantitative descriptive - correlational. The unit of analysis were 60 patients hospitalized in the Clínica San Juan de Dios, the information was generated through two instruments, a survey implemented by the professional staff of caregivers and nurses for a checklist performed by nursing professionals, was performed according to time of occurrence prospectively. Results: $67.5 \%$ of family caregiver skills deficient level presents accompanied by an intense overload of 73\%. Conclusions: We found significant inverse relationship between the basic care skills and poor level greater burden of caregivers of patients with neurological deficit in the service of the Clinical Hospital San Juan de Dios, according to the Pearson correlation test modified, with $p=0.028$.
\end{abstract}

Keywords: Basic care skills-familiar caregiver burden in adult patients' neurological deficit.

*Correspondencia: sandrita02_8@hotmail.com,51-1-989156871 


\section{INTRODUCCIÓN}

Las enfermedades neurológicas representan un grupo de alteraciones con importantes repercusiones sociales y económicas; el costo económico relacionado con los medicamentos, cuidadores y terapeutas es alto, asimismo, el fuerte impacto que causan en las familias. La importancia de las afecciones en mención radica en la alta recurrencia, el impacto sobre la calidad de vida, la discapacidad que produce y su alto índice de mortalidad (Uribe 1997).

Según la Organización Mundial de la Salud, solo en el año 2001, 5,5 millones de personas fallecieron a consecuencia de los enfermedades cerebrales vasculares; de cada cien pacientes, diez fallecen de inmediato, quince en el transcurso del primer año y ocho en el transcurso del segundo año; es decir, $33 \%$ fallecen dentro de los dos primeros años. En Europa se produce el $49 \%$ de todas las muertes y en Estados Unidos aproximadamente doscientos mil fallecimientos anuales. En Colombia, el 28\% de todas las muertes se deben a ECV, con una incidencia de 7,2 por cada cien mil habitantes. Es la segunda causa de años de vida potencialmente perdidos y la quinta causa de años potencialmente saludables perdidos.

Nkongho (1996) plantea que la habilidad de cuidado es el potencial que tiene la persona adulta, incluyendo una dimensión cognoscitiva y otra actitudinal, que pueden evaluarse a través de la comunicación directa con cada persona. La habilidad de cuidado requiere de una relación de compromiso entre el cuidador y el receptor de cuidado, para interactuar y comprender la habilidad como una forma de vivir y no como una tarea colateral. Las habilidades de cuidado incluyen una dimensión cognitiva y actitudinal, identificadas y medidas por las dimensiones de conocimiento, valor y paciencia.

El número de personas con Síndrome de Carga del Cuidador es aún más desconocido, puesto que su rol todavía no ha sido bien identificado en la población general y su prevalencia es directamente proporcional al número de pacientes con daño cerebral; sin embargo, se conoce que la prevalencia en una población de cuidadores en Cali, es del 47\% (Florez, 1997).

En este sentido, se estima que alrededor de un $40 \%$ de los cuidadores no recibe ayuda de ninguna otra persona, ni siquiera de familiares cercanos. Sin embargo también es cierto que tienden a rechazar el apoyo exterior aun necesitándolo, algunas veces por sentimientos de culpa u obligación moral mal entendida. El uso de recursos institucionales es muy bajo ya que solo en el $15 \%$ de las familias existe apoyo municipal o autonómico de servicios sociales (Flórez, 1997).
En el Perú, hasta el momento no se conocen estudios que describan las habilidades de cuidado de los cuidadores de pacientes con secuelas neurológicas ni la carga del mismo. La observación del incremento de deficiencias neurológicas, el aumento de cuidadores familiares, en el área de hospitalización de adultos de la Clínica San Juan de Dios, con expresiones de fatiga y temor cuando estaba próxima el alta del paciente, motivó la preocupación por conocer qué tan bien sabían realizar el cuidado básico del paciente e indagar si en ellos existía la carga del cuidador.

El objetivo principal del estudio consistió en determinar la relación entre las habilidades de cuidado básico y la carga del cuidador familiar de pacientes con déficit neurológico en el servicio de hospitalización adulto mayor de la Clínica Hogar San Juan de Dios. Lima, enero a marzo del 2012.

\section{MATERIALES Y MÉTODOS}

El estudio fue observacional porque ambas variables se estudiaron tal como se comportan en la realidad, sin modificar nada. Según el alcance de los resultados fue un diseño descriptivo correlacional, porque se estableció la existencia de relación entre las habilidades del cuidado básico y la existencia de carga del cuidador familiar. Según tiempo de ocurrencia de los hechos investigados, es de tipo prospectivo y según el número de mediciones realizadas, fue transversal.

Para evaluar la carga del cuidador, se utilizó la escala de sobrecarga del cuidador de Zarit (EZ), validada en nuestro medio, (coeficiente de confiabilidad de $0,71-0,85$ en validaciones internacionales), posee una consistencia interna según el Coeficiente alfa de Cronbach, de 0,91 en estudio original, y 0,85-0,93 en varios países; ampliamente utilizada no solo en estudios de dependencia, sino también en otras poblaciones.

Consta de 22 preguntas con 5 posibles respuestas (nunca, rara vez, algunas veces, bastantes veces, casi siempre), que puntúan de 1 a 5 , y con un rango de 22 a 110 en la puntuación total, y que establece los diferentes grados de sobrecarga en función de la puntuación obtenida: ausencia de sobrecarga $(\leq 46)$, sobrecarga ligera (47-55) y sobrecarga intensa $(\geq 56)$.

El principal inconveniente para su utilización es el tamaño y, por tanto, el tiempo que se emplea en su uso.

Para evaluar la habilidad del cuidado se utilizó una lista de verificación el cual tiene tres dimensiones los cuales son: aspecto físico, apoyo psico-emocional y actitudes y valores teniendo 42 ítems. La cual fue 
diseñada por las investigadoras y validada mediante juicio de expertos y con una confiabilidad según el coeficiente alfa de Cronbach, de 0.78 .

Recolectados los datos se transfirieron a una matriz del Paquete Estadístico para las Ciencias Sociales (SPSS), procesando los resultados en tablas de contingencia de doble entrada; el nivel de significancia de los resultados fue 0.028 , significativo.

\section{RESULTADOS}

La muestra de cuidadores fue a predominio del sexo femenino $(85 \%)$, con ocupación preferencial en el hogar (50\%). El vínculo de vínculo mayor, correspondió al de esposo(a), en un $40 \%$. El número de horas dedicadas a su labor cuidadora fue entre 21 a 24 horas en un $33.3 \%$ y el grado de instrucción secundaria fue del $48.3 \%$.

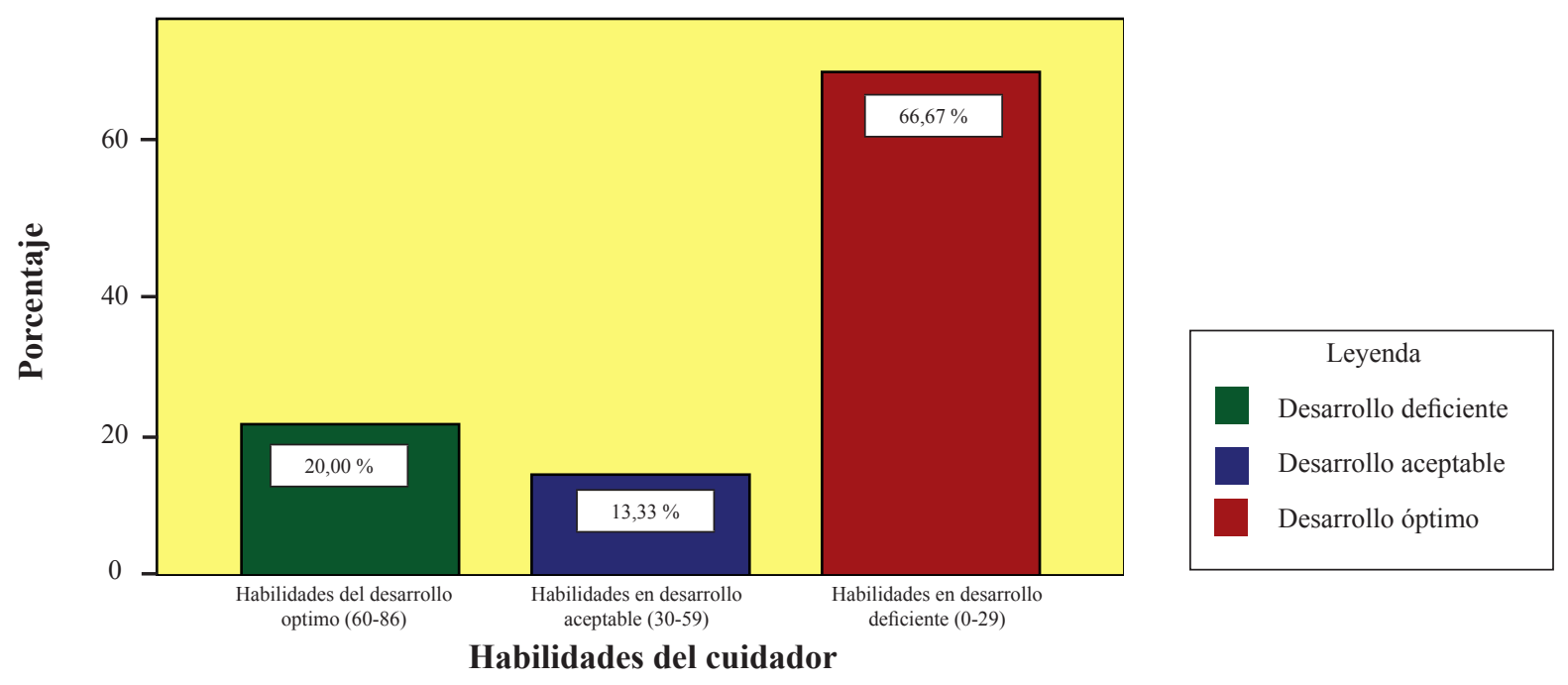

Figura 1.

Cuidador familiar del paciente con secuelas neurológicas según nivel de habilidad del cuidado básico en el Hogar Clínica San Juan de Dios, Lima -2012.

En la figura 1 se puede apreciar que del total de cuidadores estudiados, el $66.6 \%$ evidenció habilidades

deficientes de cuidado básico, los otros niveles de habilidad fueron inferiores.

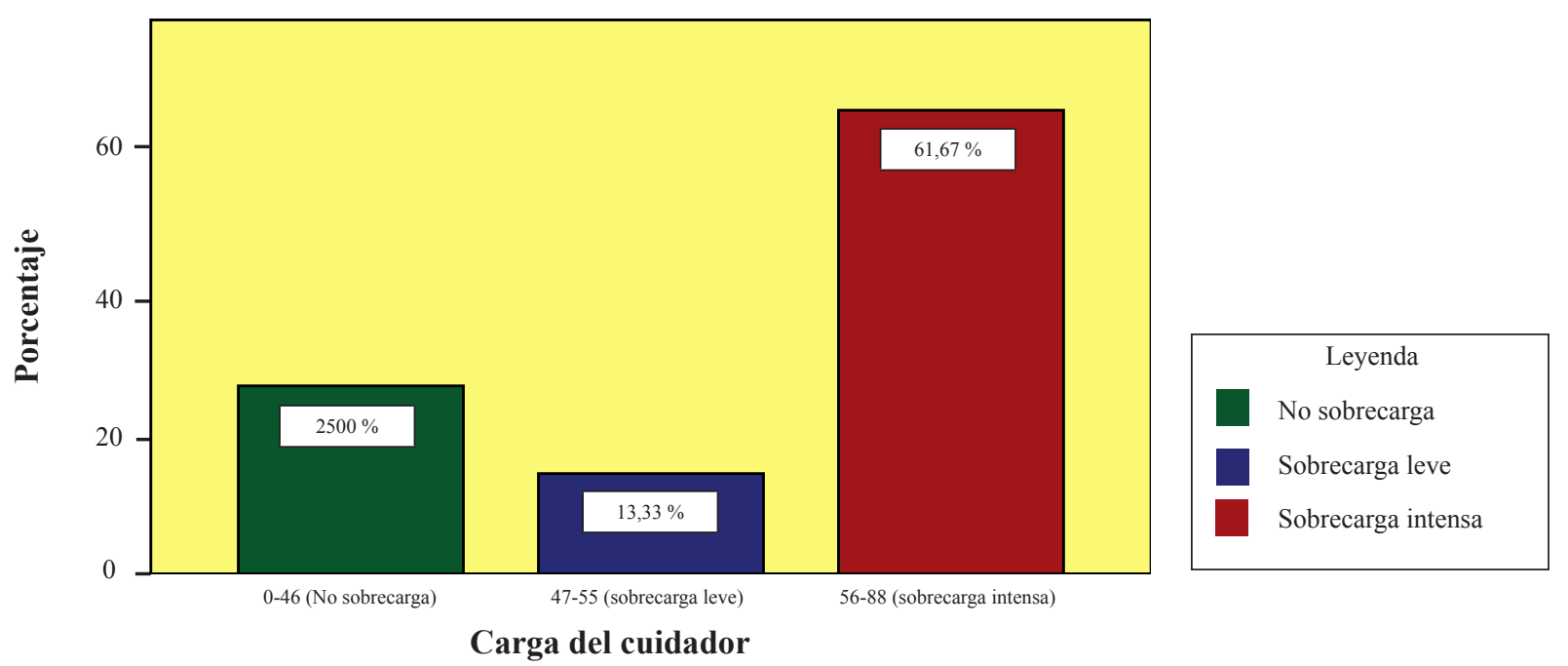

Figura 2.

Cuidadores familiares del paciente con secuela neurológica según nivel de carga del cuidador en el Hogar Clínica San Juan De Dios, Lima 2012. 
En la figura 2 se aprecia que del total de cuidadores de la muestra, el $61.67 \%$ presentó carga del cuidador en el nivel de sobrecarga intensa, con porcentajes bajos en los otros niveles.

Tabla 1

Relación entre habilidades de desarrollo y carga del cuidador de pacientes neurológicos en el Hogar Clínica San Juan De Dios, Lima, 2012

\begin{tabular}{cc}
\hline Variable & Sig. asintótica (bilateral) \\
\hline $\begin{array}{c}\text { Habilidades de desarrollo y carga del } \\
\text { cuidador de pacientes neurológicos }\end{array}$ & 0,028 \\
\hline
\end{tabular}

La correlación según la prueba Chi cuadrado de Pearson fue significativa, siendo $p<0.05$ por tanto existe relación entre las habilidades de desarrollo y carga del cuidador de pacientes neurológicos en el Hogar Clínica San Juan de Dios.

\section{DISCUSIÓN}

El presente estudio obtuvo similares indicadores a lo sugeridos por Compean, Silerio, Castillo y Parra (2008), quienes encontraron niveles de intensa sobrecarga en el cuidador primario; es importante señalar que en el estudio realizado, se obtuvo el $61.7 \%$ del total de la muestra con sobrecarga intensa. El estudio de comparación no reportó relaciones, mientras que el aporte de la investigación desarrollada, sÍ vincula la existencia de sobrecarga al número de horas de cuidado brindado al familiar con déficit neurológico.

Coincidentemente con el trabajo de Moreno (2004), desde el punto de vista socio demográfico, los cuidadores son principalmente mujeres, el mayor número de cuidadores pertenece a la generación intermedia, por encontrarse en edades entre 36 y 59 años. A la luz de estos resultados, se puede afirmar la existencia de similitudes, dado que los cuidadores familiares son también principalmente mujeres en un $85 \%$ cuyas edades están comprendidas entre 31 a 40 años, en su mayoría; esta característica, representa simultáneamente grandes fortalezas y riesgos en el desempeño del rol del cuidador, ya que en esta etapa las personas se encuentran en un período de mayor productividad y las tareas de desarrollo están orientadas a la autorrealización; la permanencia prolongada al cuidado de parientes crónicamente enfermos o con discapacidades, limita considerablemente las opciones de desarrollo personal, convirtiéndose en una fuente potencial de carga psicoemocional y social.

Parra y Berdejo (2008) encontraron, entre sus hallazgos, que la mayor proporción de cuidadores familiares son mujeres $(81,57 \%)$, siendo cuatro veces mayor que los hombres $(18,57 \%)$ y siendo el parentesco de estos cuidadores, la consanguinidad muy cercana: hijo, padre, madre, como de filiación: esposa, nuera, situación que ratifica a la familia como pilar básico de la administración de cuidado. Bustamante (2006) argumenta que desde el interior de la vida familiar y desde las etapas más tempranas de la vida, los miembros de la familia aprenden que nadie mejor que la familia puede apoyar a un ser querido cuando la salud se pierde, nadie con más conocimiento de los gustos y preferencias y a la vez nadie mejor para aceptar y/o sobrellevar los efectos de las carencias o las limitaciones biofísicas y sicosociales.

El análisis de la información encontrada, permite comprender que las habilidades poco desarrolladas, para el cuidado en los cuidadores familiares de personas discapacitadas, especialmente en la capacidad de relacionarse con el receptor del cuidado en cuanto a paciencia, confianza, coraje, sensación de pertenencia; revela deficiencias en el rol de cuidadores, el riesgo de abandono del cuidado y la desesperanza, conducen a que la calidad del cuidado que ofrecen a sus familiares discapacitados sea baja.

En el estudio queda demostrado que la mayoría de los cuidadores familiares no cuentan con la habilidad requerida para la prestación de cuidado en términos del conocimiento, paciencia y valor.

El estudio realizado, evidencia diferencias con numerosos estudios realizados en Latinoamérica y en especial en Colombia, ninguno de ellos ha medido las habilidades del cuidado básico del cuidador en las dimensiones físicas, emocionales y sociales; la metodología seguida permite ahondar en la valoración de las competencias de los cuidadores familiares muy necesarias e indispensables, para un creciente número de pacientes que se ven afectados por los efectos de las enfermedades neurológicas y sus secuelas.

Efectivamente, el estudio de cada dimensión, permite identificar necesidades específicas de intervención 
formativa dirigida a los cuidadores familiares, antes del regreso de los pacientes al hogar; según Orem (citada por Marriner, 1996), es una magnífica oportunidad para elevar el potencial de autocuidado de las personas con quienes la enfermera interactúa, mientras el paciente permanece hospitalizado.

A su vez, los hallazgos del presente trabajo, dejan entrever la necesidad de fortalecer las estrategias de enseñanza aprendizaje, en el campo extendido de la enfermería familiar, creando mecanismos que articulan progresivamente, y de modo duradero, la continuidad del cuidado entre el hospital y el hogar (Leininger citada por Marriner, 1996).

Contar además con la información específica de los criterios donde es necesario educar al cuidador familiar y la dinámica interactiva de la actividad orientadora, es una oportunidad inherente al estudio realizado.

Con respecto a diversos estudios mencionados, casi todos utilizaron la escala de Nkongho que esta subdividida en dimensiones tales como conocimiento, paciencia y valor. Para realizar el presente estudio se evaluó dicha escala la cual se llegó a la conclusión que no está acorde con las necesidades de cuidado del paciente con afecciones neurológicas, por lo que se elaboró una lista de verificación que contiene criterios para la observación y valoración de las habilidades del cuidador teniendo en cuenta tres dimensiones, aspectos físicos, apoyo psicoemocional, actitudes y valores, indispensables cuando se trata de cuidar seres humanos, instrumento que permitió identificar puntos críticos en el desempeño del cuidador familiar y de donde emergen propuestas de orden inmediato.

\section{CONCLUSIONES}

El nivel de habilidades del cuidador familiar que asiste a pacientes con déficit neurológico, fue deficiente en un $66.7 \%$, en el cuidado básico de pacientes con déficit neurológico presentando sobrecarga intensa en un $73 \%$. Se encontró relación inversa y significativa entre las habilidades del cuidador familiar y la carga del cuidador familiar, expresada en que a menor nivel de habilidades en el cuidado básico, más intensa es la sobrecarga que experimenta el cuidador familiar de pacientes con déficit neurológico en el servicio de hospitalización adultos del Hogar Clínica San Juan de Dios (valor $\mathrm{p}=0,028)$.

\section{REFERENCIAS BIBLIOGRÁFICAS}

Barrera L., Blanco L., Figueroa P., Pinto N., Sánchez B. (2006) Habilidad de cuidadores familiares de personas con enfermedad crónica: "una mirada internacional". Revista Aquichan; 6 .2. 135-144.

Barrera L., Pinto N., Sánchez B. (2006) Habilidad de cuidado de cuidadores familiares de personas con enfermedad crónica: comparación de género. Revista Actual. Enfermería. 9.2. 9-13.

Bustamente, S. (2006). Enfermería familiar. Universidad Nacional de Trujillo. Perú.

Compean G., Silerio V., Castillo D., Parra D. (2008) Perfil y sobrecarga del cuidador primario del paciente adulto mayor con afectaciones neurológicas. Revista Conaived. 13.1.17-21

Domínguez A., 2003, La percepción del cuidar. Revista. Rol de enfermería Bogotá 127.27.

Florez Lozano (1997). Envejecimiento, perso-nalidad y deterioro cognitivo: manejo del anciano en atención primaria. Barcelona: Edika-Med, 1996. ISBN 847877-166-2.

Marriner Tomey (1996). Modelos y teorías de enfermería. Ed. Interamericano. México.

Moreno M., Nader A., López C. (2004). Relación entre las características de los cuidadores de familias de pacientes con discapacidad y la percepción de $\mathrm{su}$ estado de salud. Revista Avances de Enfermería, Vol. XXII No. 1, 12-14.

Nkongho N. (1996). Elaboración de sistemas de cuidado en las familias áfrica americanos. Applied Nursing Research; 9 (3):108-14.

Parra y Berdejo. (2008) Habilidades para el cuidado y depresión de cuidadores de pacientes con demencia. Revista Colombiana de Psicología. 15 -17.

Uribe C., Jiménez I., Mora M., Arana A., Sánchez J., Zuluaga L. (1997) Epidemiología de las enfermedades cerebrovasculares en Sabaneta, Colombia. Revista Neurológica. 25.1008-1012. 